\title{
Locating Motion Artifacts in Parametric fMRI Analysis
}

\author{
A.J. Lacey, N.A. Thacker, E. Burton, and A. Jackson \\ Division of Imaging Science and Bio-medical Engineering \\ University of Manchester, Manchester, M13 9PT \\ a. lacey@man.ac.uk \\ http://www.niac.man.ac.uk
}

\begin{abstract}
In this paper we assess rigid body co-registration in terms of residual motion artifacts for the different correlation approaches used in fMRI. We summarise, from a statistical perspective, the three main approaches to parametric fMRI analysis and then present a new way of visualising motion effects in correlation analysis. This technique can be used both to select regions of relatively unambiguous activation and to verify the results of analysis. We demonstrate the usefulness of this visualisation technique on fMRI data sets suffering from motion correlated artifacts. We use it in our assesment of rigid body co-registration concluding that it is an acceptable basis for re-alignment, provided that correlation is done using a measure which estimates variance from the data at each voxel.
\end{abstract}

\section{Introduction}

Subject motion during the time course of functional activation studies has been shown to cause spurious signals which can mimic "true" activation [1,2]. Motion correction using image registration software is therefore common practice in functional imaging and many groups now routinely make use of rigid body co-registration using software packages such as Automatic Image Registration (AIR) [3,4,5]. However, depending on the details of the data capture, the assumption of a rigid body may be inappropriate. In some cases slices of image data may undergo different amounts of motion and in other cases the data may suffer from motion blurring. These processes could leave residual motion artifacts in the data which bias subsequent interpretation.

Previous workers have demonstrated the potential problems of motion in fMRI analysis $[1,2]$ using data sets with simulated movement. The estimation of this effect shows the likely scale of this contribution to an fMRI signal. We have found no paper which attempts to quantify directly the affect on the computed correlation measures.

The objective of this work was to develop a technique to test whether rigid body co-registration successfully removes motion effects to a statistically acceptable level. We first review the statistical characteristics of published analysis techniques in order to design a test for the effectiveness of rigid-body coregistration which is applicable to the range of current approaches. We then

C. Taylor, A. Colchester (Eds.): MICCAI'99, LNCS 1679, pp. 524-532, 1999.

(C) Springer-Verlag Berlin Heidelberg 1999 
go on to demonstrate the effects of motion and co-registration on the common statistical correlation forms used for analysis.

\section{2 fMRI Analysis Techniques}

There have been many approaches to the analysis of functional NMR images proposed in the literature. Considered from a statistical point of view these techniques can be grouped as either non-parametric or parametric. It is generally accepted that whilst non-parametric techniques are initially more robust, parametric techniques will ultimately have better discriminability once the analytical models have been refined. Generally, parametric analysis can be decomposed into two stages; the application of a voxel by voxel time dependent analysis, followed by a regional analysis of clusters [6,7]. The first of these is designed as a significance test, the hypothesis being that the data seen in the image can be accounted for entirely by random noise fluctuations. The second is a significance test based on the probability of observing particular sized regions failing the first test. This process is well suited to removing isolated "fake" activations but doesn't perform well on larger connected regions such as those often presented by motion. Thus it is the performance of the first test with which we are concerned.

The voxel based null hypothesis test is generally implemented as a correlation measure between the image signal and a normalised wave-function which models the activation paradigm. The details of this correlation measure vary in the literature, but all successful measures have the same fundamental statistical origins; some measure of correlation $C$ is normalised by it's expected variance $\operatorname{var}(C)$ in order to produce a measure which can be treated like a 't test' or 'Z score' [7]. As regards the selection of a wave-function, although the ability to extract signal will be strongly dependent on having the correct functional form and phase, the technique of error propagation can be used to show that any normalised wave-function will produce a set of correlation measures with an identical distribution for the null hypothesis. Thus we choose to work with a square wave for simplicity.

Three measures, chosen to cover a set of statistically distinct possible approaches, were used for this investigation;

$$
C_{j}^{1}=\sum_{t=1}^{T} I_{t j} . W_{t}
$$

A simple correlation measure with no explicit (fixed) normalisation, where $W$ is a normalised correlation waveform $\left(|W|^{2}=1\right)$ and $I_{j}$ is a mean subtracted temporal data set at voxel $j$. This correlation measure can be converted into a simple null hypothesis statistic by dividing by a pooled estimate of the standard deviation on the measure $\sqrt{\operatorname{var}(C)}$ and will behave in the same way as any measure which makes the basic assumption of constant uniform image noise, including Fourier approaches. While we accept that such a simple measure is unlikely to be used unmodified in serious fMRI analysis we have included it here for completeness. 


$$
C_{j}^{2}=\frac{\sum_{t=1}^{T} I_{t j} \cdot W_{t}}{1 /(T-1) \sqrt{\sum_{t}^{T}\left(I_{t j}-W_{t} \cdot C_{j}^{1}\right)^{2}}}
$$

A measure with individual voxel variance estimation which we believe to be statistically equivalent to that used in SPM [8], where the numerator is the estimate of variation about the assumed model. This technique will behave in the same way as any measure which estimates variance from the data, such as 't-tests' and 'z-scores'.

$$
C_{j}^{3}=\frac{\sum_{t=1}^{T} I_{t j} . W_{t}}{\sqrt{\sum_{t}^{T} I_{t j}^{2}}}
$$

A normalised correlation measure as used in STIMULATE [9]. This measure is normalised between -1 and 1 but cannot be interpreted as a standard null hypothesis statistic unless the numerator approaches that of $C_{j}^{2}$, which will only happen when the noise dominates the observed signal distribution (i.e. $C_{j}^{1}$ is small).

\section{Experiments}

Experiments were performed on 6 healthy volunteers. Volunteers A,B and C were scanned at rest and volunteers $\mathrm{D}, \mathrm{E}$ and $\mathrm{F}$ performed a motor activation paradigm (finger tapping) during scanning. Head movements were minimised using foam padding and a velcro strap and a bite bar with a groove was used to provide a reference to minimise out of plane movements. For each subject 18 dynamic acquisitions of $50 \times 3 \mathrm{~mm}$ contiguous transverse slices covering the whole head using a $\mathrm{T} 2 *$-weighted gradient echo sequence were acquired.

Our investigation involves the following steps:

- Calculate rigid body motion for genuine null hypothesis data (no activation) for two groups of three subjects. One group performing the motion stimulus paradigm and the others at rest. All data were registered to a base volume for each volunteer using the Woods algorithm [3,4]. The main automated image registration (AIR) parameters were set as follows: intensity threshold $=275$, initial sampling interval $=81$, final sampling interval $=1$ pixel, sample increment decrement ratio $=3$, convergence criteria $=0.0005$, maximum number of iterations for each sampling density $=300$, no spatial smoothing was used, interpolation to standard voxel size was active.

- Compute simulated data from the motion vectors.

The motion parameters were used to interpolate a set of data from the first volume with equivalent motion vectors to those estimated by the AIR package. 
- Demonstrate the effects of motion on the correlation measures used in real data.

As the effects of motion are expected to be proportional to local image gradient, we plot correlation scores against this quantity. Here we define image gradient $G_{j}$ as follows;

$$
\begin{gathered}
d I_{x, y} / d x=\left(I_{x+1}^{\prime}-I_{x-1}^{\prime}\right) / 2 \\
d I_{x, y} / d y=\left(I_{y+1}^{\prime}-I_{y-1}^{\prime}\right) / 2 \\
G_{j}=\sqrt{\left(\left(d I_{j} / d x\right)^{2}+\left(d I_{j} / d y\right)^{2}\right)}
\end{gathered}
$$

where $x$ and $y$ are image indices and $I^{\prime}$ is the first input image of the temporal sequence smoothed with a unit Gaussian kernel. The smoothing process sets the scale for the range of applicability of the linear assumption and allows the gradient information to be estimated from a single temporal slice. Averaging of the temporal data set to produce a mean gradient would also be possible and perhaps even preferable for very large motions, but was not found necessary for this work.

For data without motion artifact, this plot is expected to show the correlation score, distributed normally around zero correlation with variable density along the image gradient axis. For this case a fixed threshold value will have the same affect in terms of rejecting the null hypothesis for all values of image gradient. Any variation from this distribution shows itself as increased broadening or non-ideal structure perpendicular to the image gradient axis.

- Demonstrate that these effects are also visible in the simulated data.

The simulated data, being effectively noise free, does not yield sensible variance estimates in the denominator terms of the standard correlation measures, $C^{2} \& C^{3}$. However, the individual estimates of the denominators are expected to be quite constant across the dataset. As a consequence $C^{1}$ is used to analyse the simulated data as it is expected to behave in a very similar fashion to the other two measures.

- Demonstrate that these effects are removed by rigid body coregistration in the real data by repeating the analysis for motion corrected data.

- Simulate the effect of isolated failures of co-registration by shifting an image in each co-registered sequence.

\section{Results and Discussion}

The standard deviations on the estimated motions are shown in figure 1 for the two groups of three subjects (A,B,C and D,E,F). For the group with no stimulus these results are close to the expected accuracy of the AIR software and the motion scatter plots, figure 2(a), show no unexpected structure or observable correlation between edge contrast and the correlation score for any of the three 


\begin{tabular}{|c|c|c|c|c|c|c|}
\hline & Pitch & Roll & Yaw & $\mathrm{X}$ & $\mathrm{Y}$ & $\mathrm{Z}$ \\
\hline $\mathrm{A}$ & $0.20^{\circ}$ & $0.22^{\circ}$ & $0.30^{\circ}$ & 0.49 pixels & 0.31 pixels & 0.06 pixels \\
$\mathrm{B}$ & $0.22^{\circ}$ & $0.49^{\circ}$ & $0.16^{\circ}$ & 0.25 pixels & 0.50 pixels & 0.11 pixels \\
$\mathrm{C}$ & $0.22^{\circ}$ & $0.33^{\circ}$ & $0.18^{\circ}$ & 0.28 pixels & 0.30 pixels & 0.09 pixels \\
$\mathrm{D}$ & $0.90^{\circ}$ & $0.60^{\circ}$ & $0.38^{\circ}$ & 0.96 pixels & 0.84 pixels & 0.31 pixels \\
$\mathrm{E}$ & $0.85^{\circ}$ & $0.06^{\circ}$ & $0.41^{\circ}$ & 0.45 pixels & 0.57 pixels & 0.16 pixels \\
$\mathrm{F}$ & $0.39^{\circ}$ & $0.12^{\circ}$ & $0.37^{\circ}$ & 0.37 pixels & 0.41 pixels & 0.17 pixels \\
\hline
\end{tabular}

Fig. 1. Standard deviations on estimated movement for each subject calculated using AIR

measures. In contrast subjects D, E \& F show significant movement, figure 1, and generate plots with distinct structure, figure $2(\mathrm{~b})$. Notice the broadening of the data in the $y$-dimension (correlation score) towards the end of the $x$-axis where the image gradient is largest (at edges).

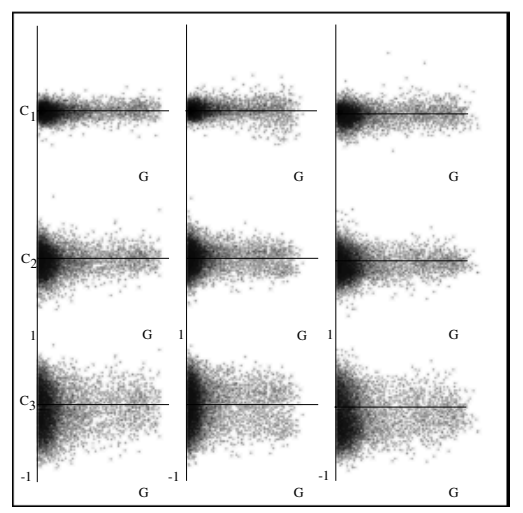

(a) No stimulus subjects A, B and $\mathrm{C}$ (columns)

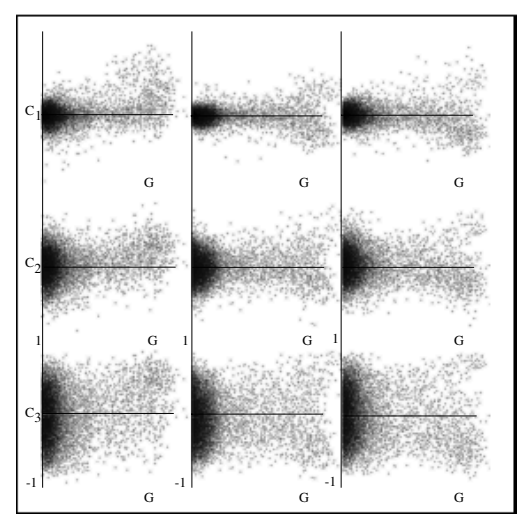

(b) Motion stimulus subjects D, $\mathrm{E}$ and $\mathrm{F}$ (columns)

Fig. 2. Correlation scores v edge contrast $(G)$ plots. Correlation scores are rows $C^{1}, C^{2}, C^{3}$ from top to bottom

Applying motion vectors estimated using the AIR software to the genuine null hypothesis data (no activation) gives the plots of figure 3(a). The distributions we see in these plots are entirely consistent with rigid body motion plus random noise. In contrast the plots of co-registered data, figure 3(b), show that this correlation has been significantly reduced by the process of co-registration. $C^{1}$ shows a gradual increasing instability (broadening) of the correlation score with edge contrast when compared to $C^{2}$ and $C^{3} \cdot C^{3}$ is marginally less affected by motion than the conventional null hypothesis statistic because the denominator 
term is increased by the variation in the image data induced by motion. While $C^{3}$ may be adequate for visually identifying relative large measures, the lack of a meaningful scaling makes this correlation score more difficult to interpret.

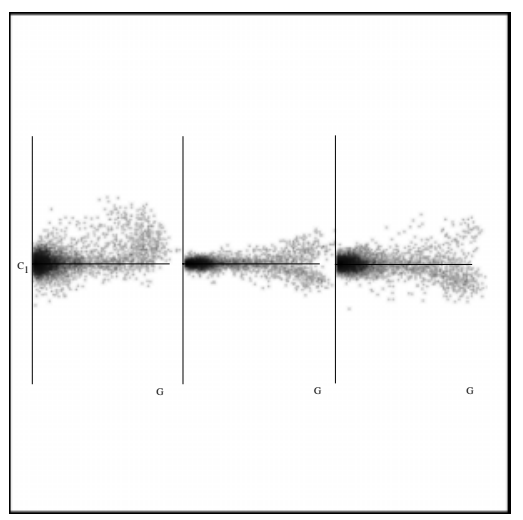

(a) Simulated stimulus

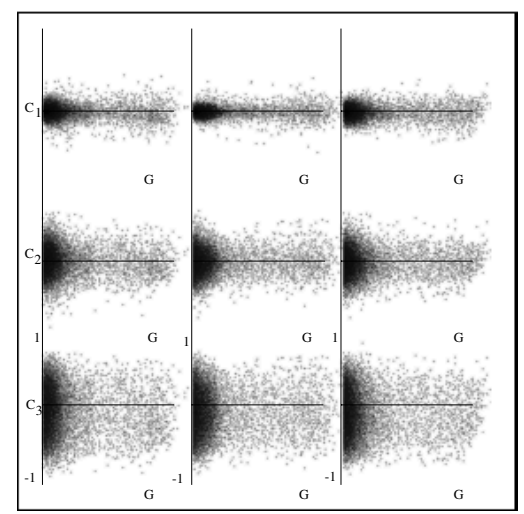

(b) Motion corrected stimulus

Fig. 3. Correlation scores v edge contrast $(G)$ plots for subjects D, E and F (columns). Correlation scores are rows $C^{1}, C^{2}, C^{3}$ from top to bottom (only $C^{1}$ is shown in the left plot)

The majority of problems occur when motion artifacts mimic the effects of signal by correlating with the stimulus response function (see figure 2(b)). No motion correction procedure can be perfectly accurate and some residual errors are to be expected. This can be observed after motion correction by calculating the ratio of variances for the correlation measures for two ranges of image gradient, figure 4(a). The ratio of the variance is calculated between the first eighth (corresponding to brain tissue) and second eighth (corresponding to the boundary between brain tissues) of the dynamic range of image gradients ( $x$-axis). The accuracies of these ratios are of the order of $1 \%$. Figure $4(\mathrm{a})$ shows that $C^{1}$ in particular is badly affected by the increased instability around edges.

When performing rigid body alignment with an iterative automated system there is always a chance that the alignment algorithm will fail. When this happens (if it is not picked up by a validation process) it will generate isolated temporal data points at each voxel which will reduce the overall correlation scores for measures such as $C^{2}$. We can illustrate this by offsetting the first image in each sequence of data by two pixels and recomputing the variances for gradients corresponding to tissue boundaries, figure 4(b). In general, any motion correction technique, even a poor one, removing the majority of the motion from the data set and leaving only residual behaviour which is uncorrelated with the stimulus response curve, will not invalidate (statistically) the conclusions of any study using such a measure. Thus our results suggest that rigid body co-registration does 


\begin{tabular}{|c|c|c|c|}
\hline Subject & $\mathrm{D}$ & $\mathrm{E}$ & $\mathrm{F}$ \\
\hline$C^{1}$ & 1.27 & 1.21 & 1.13 \\
$C^{2}$ & 1.00 & 0.98 & 1.05 \\
$C^{3}$ & 1.01 & 1.03 & 1.04 \\
\hline
\end{tabular}

(a) Intermediate and low gradient correlations

\begin{tabular}{|c|c|c|c|}
\hline Subject & $\mathrm{D}$ & $\mathrm{E}$ & $\mathrm{F}$ \\
\hline$C^{1}$ & 1.31 & 1.58 & 1.21 \\
$C^{2}$ & 0.96 & 0.99 & 0.93 \\
$C^{3}$ & 0.96 & 0.98 & 0.95 \\
\hline
\end{tabular}

(b) Intermediate gradient correlations for offset data

Fig. 4. Dimensionless measures of relative standard deviation $\left(\sqrt{\operatorname{var}\left(C_{1 / 8}\right) / \operatorname{var}\left(C_{0 / 8}\right)}\right)$

effectively remove false correlations caused by motion correlated to the stimulus response.

\section{Use of Motion Correlation Plots in fMRI Quality Control}

Figure 5(a) shows a motion correlation plot for data generated during analysis $\left(C^{2}\right)$. Activations are present in this data at both high and low level image gradient. In itself this would not be unduly worrying, but there is also a general broadening of the underlying distribution for positive correlation values at high gradient. On closer inspection this data set was found to have systematic shift artifacts at the level of 0.5 pixels in almost half of the re-sliced data set due to failure of the automatic co-registration software. The manually selected region of relatively unambiguous activation is shown as a rectangle. The new analysis technique allowed us not only to identify genuine activation, figure 5(b) but also perform quality control on our software analysis chain.

\section{Conclusions}

We have analysed the affect of motion on fMRI analysis. Given the broad range of approaches in the literature we have concentrated this analysis on the common statistical foundations of the parametric methods, which are the use of correlation measures. Our results are expected to be independent of the details of the shape of the correlation stimulus and should generalise to all fMRI studies based on assessing levels of significance from correlation scores.

We have constructed a method for visualising the effects of motion on fMRI analysis. As the effects of motion are expected to be proportional to the local image gradient, a scatter plot of correlation function versus image gradient separates the effects of motion across the plot. This distribution is self scaling, 


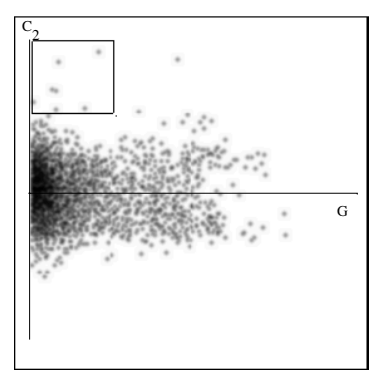

(a) $\begin{array}{llr}\text { score } C^{2} & \mathrm{v} & \text { edge } \\ \text { contrast } & & \end{array}$

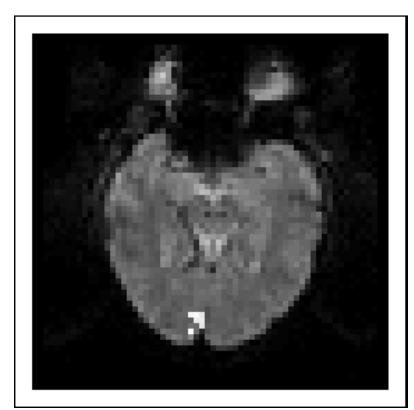

(b) fMRI image with superimposed activation

Fig. 5. Quality control of fMRI analysis showing the activation corresponding to unambiguous correlations (data within manually selected box)

easily visually interpreted and can be used as a general tool to check the relative accuracy of different motion correction procedures. Such a method could also be used with non-normalised correlation measures (i.e. $C^{1}$ ) in order to assess the adequacy of particular co-registration procedures in the absence of ground truth.

In this study we have used the new technique to visualise the effects of motion in a typical fMRI study for three correlation approaches. The results indicate that motion artifacts are manifest in motion based experiments. These effects are significantly reduced after motion correction but still observable in simple correlation analyses which assumed pooled variance. Finally, null hypothesis based correlation methods which estimate the variance from the data at each voxel are unaffected by motion provided that any resulting residual motion, is uncorrelated with the stimulus. As we see no residual correlation with the stimulus response curve following rigid body re-alignment, we consider the rigid body assumption an acceptable basis for motion correction for these measures. In general, it will never be possible to remove all stimulus correlated motion completely, but techniques, such as those described here, could be used to monitor the success or failure of attempts to do so.

Our TINA software system is available as open source, including the algorithms discussed in this paper, from http://www.niac.man.ac.uk/Tina 


\section{References}

1. J V Hajnal, I R Young, and G M Bydder. Contrast mechanisms in functional mri of the brain. In W G Bradley and G M Bydder, editors, Advanced MR Imaging Techniques, pages 195-207. Martin Dunitz Ltd London, 1997. 524

2. K J Friston, J Ashbuner, C D Frith, J B Poline, J D Heather, and R S J Frackowiak. Spatial registration and renormalisation of images. M.R.M., 2:165-188, 1995. 524

3. R P Woods, S R Cherry, and J C Mazziotta. Rapid automated algorithm for aligning and reslicing pet images. JCAT, 16:620-633, $1992.524,526$

4. R P Woods, J C Mazziotta, and S R Cherry. Mri-pet registration with an automated algorithm. JCAT, 17:536-546, 1993. 524, 526

5. A Jiang. Motion detection and correction in functional $\mathrm{mr}$ imaging. H.B.M., 3:224-235, 1995. 524

6. K J Friston, A Holmes, J B Poline, C J Price, and C D Frith. Detecting activations in pet and fmri: Levels of inference and power. Neuroimage, 40:223-235, 1996. 525

7. N Lange. Tutorial in biostatistics, statistical approaches to human brain mapping by functional magnetic resonance imaging. Statistics in Medicine, 15:389-428, 1996. 525

8. K J Friston, R J Dolan, and R S J Fackowiak. Statistical parametric mapping, 1991. MRC Cyclotron Unit, Hammersmith Hospital, London, England. 526

9. J P Strupp. Stimulate: A gui based fmri analysis software package. Neuroimage, 3, 1996. 526 